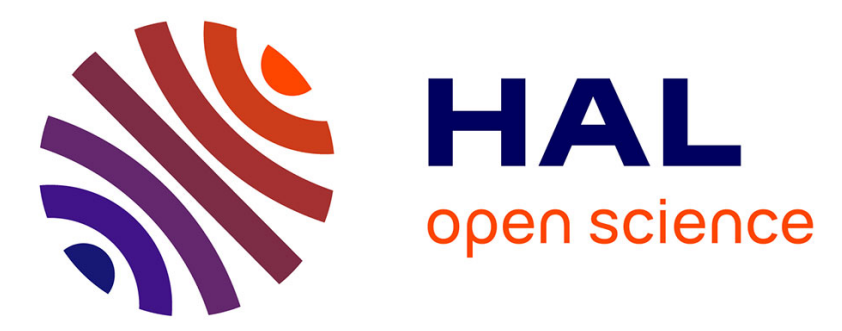

\title{
Réalisation et propriétés des photopiles solaires en couches minces de tellurure de cuivre et tellurure de cadmium
}

\author{
J. Lebrun
}

\section{- To cite this version:}

J. Lebrun. Réalisation et propriétés des photopiles solaires en couches minces de tellurure de cuivre et tellurure de cadmium. Revue de Physique Appliquée, 1966, 1 (3), pp.204-208. 10.1051/rphysap:0196600103020400 . jpa-00242717

\section{HAL Id: jpa-00242717 https://hal.science/jpa-00242717}

Submitted on 1 Jan 1966

HAL is a multi-disciplinary open access archive for the deposit and dissemination of scientific research documents, whether they are published or not. The documents may come from teaching and research institutions in France or abroad, or from public or private research centers.
L'archive ouverte pluridisciplinaire HAL, est destinée au dépôt et à la diffusion de documents scientifiques de niveau recherche, publiés ou non, émanant des établissements d'enseignement et de recherche français ou étrangers, des laboratoires publics ou privés. 


\title{
REALISATION ET PROPRIETÉS DES PHOTOPILES SOLAIRES EN COUCHES MINCES DE TELLURURE DE CUIVRE ET TELLURURE DE CADMIUM
}

\author{
Par J. LEBRUN, \\ Radiotechnique - Coprim R. T. C., Suresnes.
}

\begin{abstract}
Résumé. - On réalise des photopiles en couches minces en déposant sur une feuille de molybdène du tellurure de cadmium $(n)$, par réaction en phase vapeur des constituants. La région $(p)$ est obtenue par évaporation sous vide de tellurure de cuivre, le contact étant assuré par une grille d'or évaporé. On a mesuré les principales caractéristiques de ces cellules. Pour un éclairement de $50 \mathrm{~mW} / \mathrm{cm}^{2}$, on obtient un courant de court-circuit de $7 \mathrm{~mA} / \mathrm{cm}^{2}$, une tension à vide de 400 à $500 \mathrm{mV}$ et un rendement énergétique de $3 \%$.
\end{abstract}

Abstract. - We have made thin film solar cells by depositing cadmium telluride on molybdenum sheet by vapor reaction of the components. The layer is obtained by vacuum evaporation of copper telluride and the $p$ contact is made by an evaporated gold grid. We have measured the parameters of these cells. For a solar energy of $50 \mathrm{~mW} / \mathrm{cm}^{2}$ the short circuit current is $7 \mathrm{~mA} / \mathrm{cm}^{2}$, the open voltage is 400 to $500 \mathrm{mV}$ and the efficiency $3 \%$.

I. Introduction. - Pour des raisons techniques et économiques les cellules solaires en couches minces présentent un grand intérêt. La présente étude sur les photopiles solaires en couches minces de tellurure de cadmium et tellurure de cuivre a été entreprise à la Radiotechnique Coprim, dans le cadre d'un contrat du Centre National d'Études Spatiales.

Le dépôt sur support de molybdène du CdTe dopé $n$ s'obtient par une méthode équivalente à celle utilisée par Cusano [1] sans dopage au gallium cepen- dant. La partie $p$ réalisée par différentes méthodes dont l'une d'elles, mise au point au C. N. R. S. à Bellevue [2] sur du CdTe massif, constitue une solu. tion originale pour le matériau en couche mince.

Il nous a alors été possible de comparer ces diverses techniques de réalisation ainsi que les pro priétés des cellules réalisées.

II. Réalisation des photopiles. - La figure 1 montre les différentes structures étudiées sur sup port de molybdène avec ou sans CdS intermédiaire

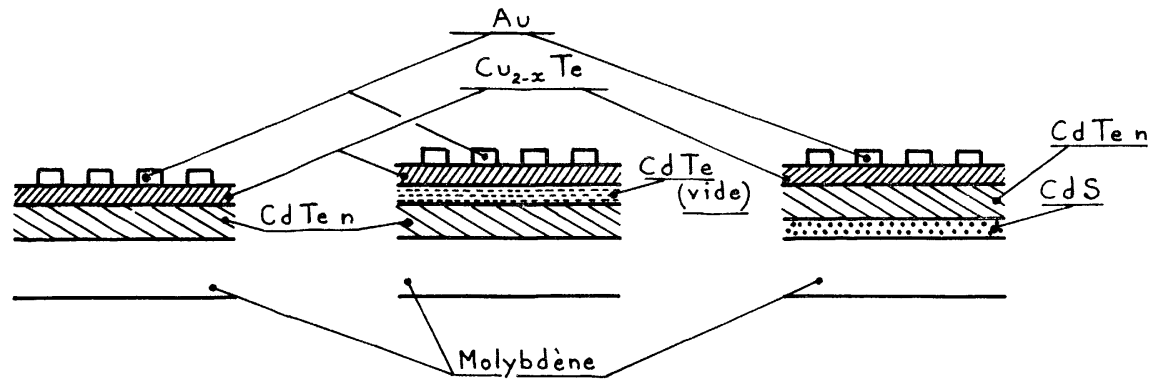

Fig. 1.

entre CdTe $(n)$ et molybdène, avec ou sans CdTe intermédiaire entre la couche $(n)$ et $(p)$. La figure 2 rappelle l'essentiel du principe de l'appareillage type Cusano.

A. Réalisation de la couche $n$. Propriétés. a) Pour la mesure des propriétés optiques ou électriques de la couche $n$, il est nécessaire d'avoir des dépôts témoins sur support pyrex. Toutefois les propriétés mesurées dans ces conditions sont des pro- priétés longitudinales alors que nous utilisons des propriétés transversales dans la photopile ; les résistivités (sans dopage au Ga) s'échelonment entre 50 et $1000 \Omega \mathrm{cm}$, les mobilités sont comprises entre 20 et $100 \mathrm{~cm}^{2} / \mathrm{V}$.s.

$b$ ) Pour les dépôts sur molybdène, s'est posé le problème du contact métal CdTe ; nous montrerons plus loin ses effets sur les caractéristiques $I-V$, à l'obscurité et sous éclairement. L'insertion d'une couche intermédiaire de $\mathrm{CdS}$ résoud en partie le 


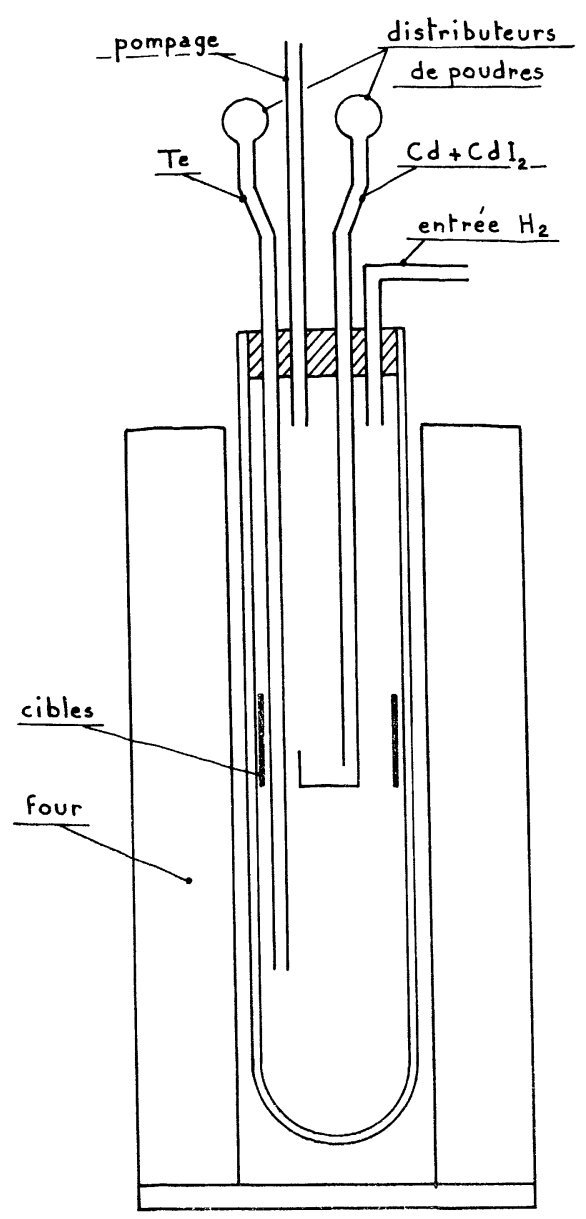

Frg. 2. - Enceinte de réaction.

problème. La granulométrie du dépôt est très variable. On note des zones cristallisées comme le montre un examen en diffraction électronique.

B. Réalisation de la couche p. Propriétés. a) Méthode par immersion. Nous avons d'abord utilisé cette méthode mentionnée dans la littérature [1] pour former une fine couche transparente de $\mathrm{Cu}_{2-x} \mathrm{Te}$.

L'étude de l'épaisseur du dépôt en fonction du temps d'immersion a été conduite en mesurant la résistance superficielle en fonction du temps. La figure 3 nous montre que, pour la solution utilisée et au bout de 15 secondes, on obtient des dépôts de $100 \Omega$ /carré. En général, nous stopnons l'immersion à cette valeur.

b) Évaporation d'une fine couche de $\mathrm{Cu}_{2-x} \mathrm{Te}$ sous vide par une méthode flash : sur des supports témoins nous pouvons mesurer les paramètres de ces dépôts. En particulier, la résistance superficielle est de l'ordre de $100 \Omega /$ carré, la mobilité de l'ordre de $6 \mathrm{~cm}^{2} / \mathrm{V}$.s, ce qui conduit à des densités de $10^{21}$ porteurs environ. La figure 4 donne la courbe de

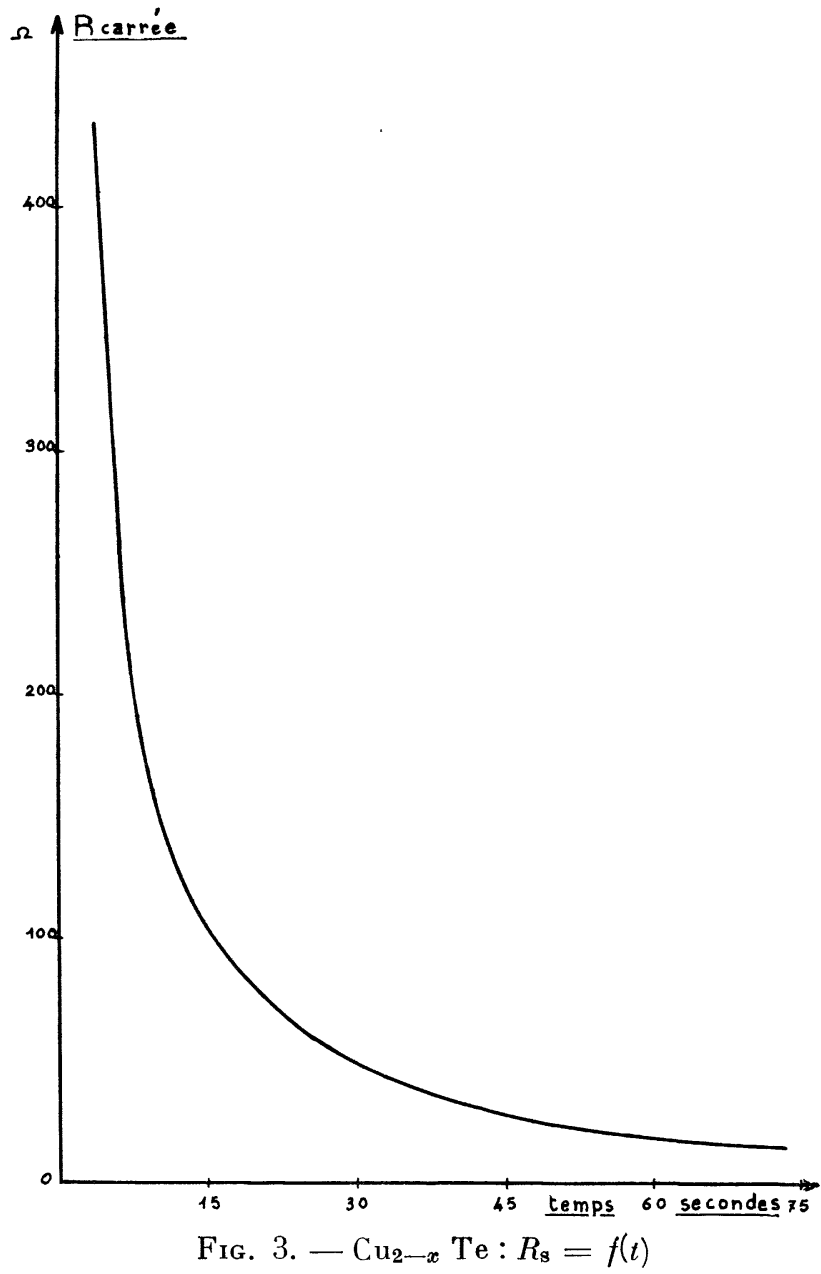

transmission optique $\mathrm{du} \mathrm{Cu}_{2-x} \mathrm{Te}$ obtenu par cette méthode.

c) Évaporation d'une couche de CdTe intrinsèque intermédiaire : dans la préparation qui s'inspire intégralement de celle décrite par Cusano, il est possible en fin de dépôt d'obtenir une région

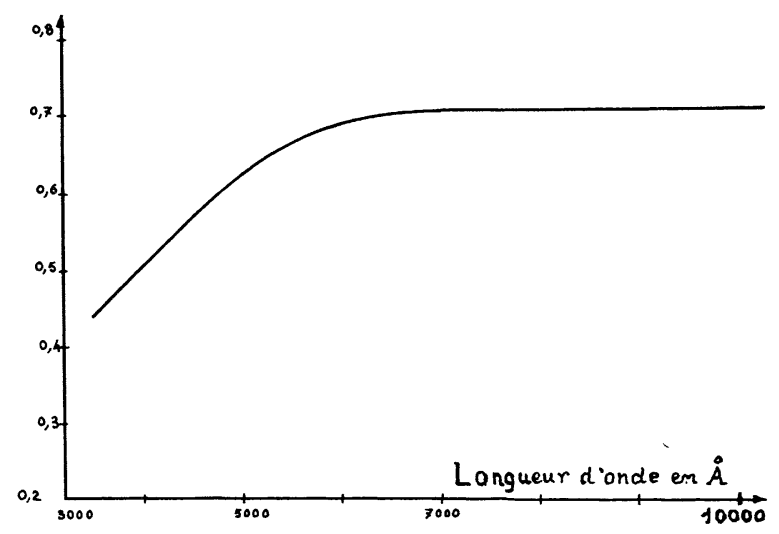

Fig. 4. - Transmission optique $\mathrm{Cu}_{2-x}$ Te. 
moins $n$ et même légèrement dopée $p$. Cette région est essentielle à l'obtention de bonnes performances photovoltaïques. Après nous être assuré qu'une telle couche de transition n'existait pas dans nos échantillons, nous avons évaporé sous vide une couche de CdTe intrinsèque d'au moins $1000 \AA$. La couche évaporée de $\mathrm{Cu}_{2-x} \mathrm{Te}$ complète alors la structure.

d) Nous avons également évaporé une fine cuuche d'or transparente sur CdTe $n$ et tenté des traitements thermiques pour obtenir un effet photovoltaïque. Nous en donnerons plus loin les résultats.

e) Enfin comme collecteur une grille d'or évaporée : sa transparence est de $80 \%$ environ. Le contact $\mathrm{Au}-\mathrm{Cu}_{2}-x \mathrm{Te}$ se révèle parfaitement ohmique et de faible valeur. Nous obtenons ainsi des résultats plus reproductibles qu'avec des grilles métalliques appliquées, les performances maximales étant identiques.

III. Propriétés des photopiles. - a) Technique d'étude d'un paramètre. - Par la réalisation de cellules jumelées sur un même support, donc présentant peu d'écart quant à la qualité de la couche $n$, il est possible d'étudier deux cellules présentant une faible différence dans un paramètre.

b) La comparaison des rendements obtenus permet l'élimination d'un"certain nombre de méthodes de réalisation. C'est ainsi qu'à qualité égale de la

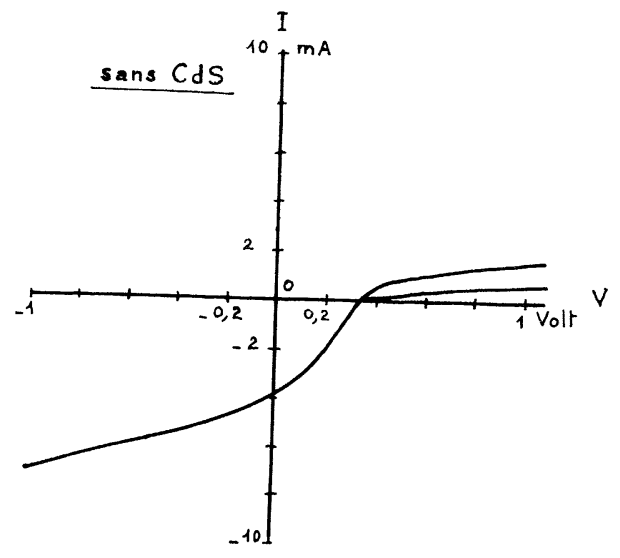

couche $n$, nous avons obtenu les rendements sui. vants :

$$
\begin{array}{ll}
\mathrm{Au} & \text { Evap. } 1 \% \text { max. } \\
\mathrm{Cu}_{2-x} \mathrm{Te} & \text { Immersion } 1,5 \% \text { max. } \\
\mathrm{Cu}_{2-x} \mathrm{Te} & \text { Évap. }
\end{array}
$$

Ces résultats sont significatifs : il est beaucoup plus sûr de réaliser des cellules de bonnes performances en évaporant le $\mathrm{Cu}_{2-x} \mathrm{Te}$.

La cellule CdTeAu n'est évidemment qu'un simple contact métal semiconducteur.

L'immersion provoque de nombreux courtscircuits et, si on peut encore obtenir sous éclairement des courants de court-circuit convenables, les tensions à vide sont toujours relativement faibles. Ces conclusions ne sont toutefois valables que pour la qualité des dépôts que nous réalisons actuellement.

En ce qui concerne les cellules avec couche $p$ obtenue par évaporation, la comparaison entre les deux méthodes de fabrication, avec et sans CdTe intrinsèque intermédiaire donne les résultats suivants, établis sur de nombreux échantillons :

Tension à vide $V_{\text {co }}$ (avec $\mathrm{CdTe}$ ) supérieure de $24 \%$.

Courant de court-circuit $I_{\mathrm{cc}}$ (avec CdTe) inférieur de $18 \%$.

c) Caractéristiques $I-V$. - Elles sont relatives aux structures avec CdTe intermédiaire.

1. La figure 5 montre comment la sous-couche

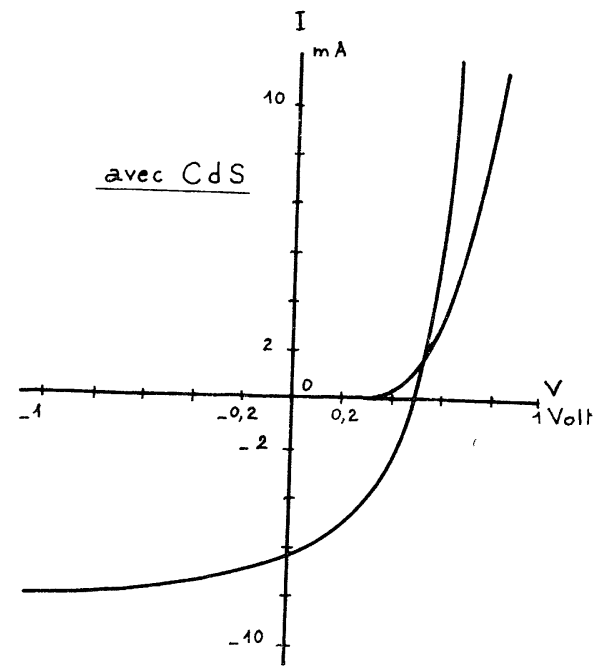

Fig. 5. - Caractéristique $I=f(V)$.

de CdS améliore ces caractéristiques en supprimant le contact redresseur Mo-CdTe $(n)$ et réduisant la résistance série.

2. La figure 6 est relative à la caractéristique I. $V$. directe à l'obscurité.

De l'expression du courant

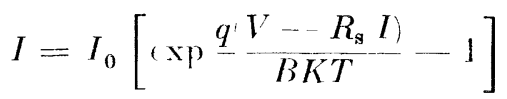

où $R_{\mathrm{s}}$ est la résistance série, on détermine :

$$
B \sim 2,7 ; \quad R_{\mathrm{s}} \sim 10 \Omega . \mathrm{cm}^{2} .
$$

3. La figure 7 nous montre les variations de $V_{\text {co }}$ et $I_{\text {cc }}$ en fonction de la puissance lumineuse reçue $W$. Le courant croît linéarrement avec la puissance reçue avec un écart de l'ordre de $10 \%$ aux forts éclairements. La tension croît proportionnellement 


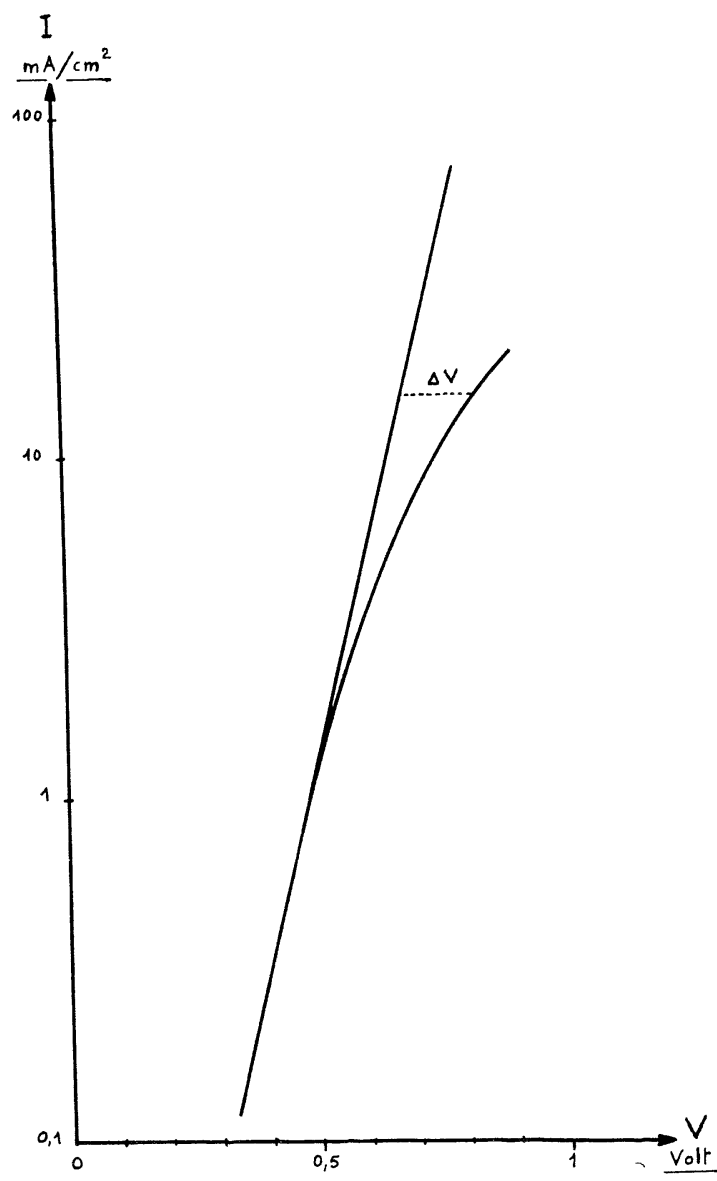

Fig. 6. - Caractéristique $I=f(V)$, obscurité.

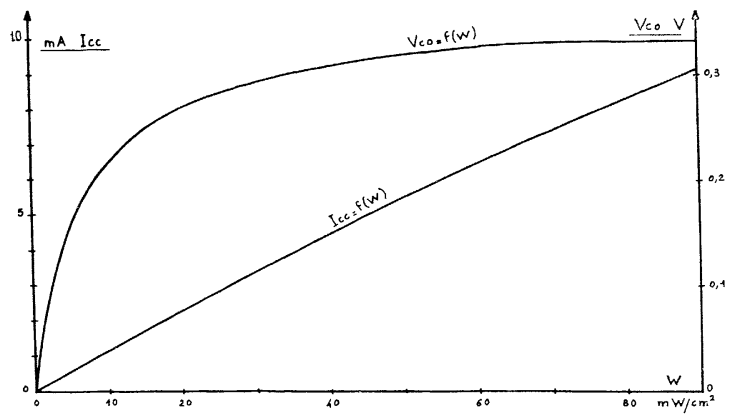

Fig. 7. - $V_{\mathrm{co}}=f(W) ; I_{\mathrm{cc}}=f(W)$.

à Log $W$ sur la figure 8 , comme le prévoit la théorie classique des homojonctions.

4. La figure 9 donne la réponse spectrale d'une cellule ; on notera la brusque chute vers $8250 \AA$.

5. Le rendement quantique déterminé à $\lambda=0,546 \mu \mathrm{m}$ et $\lambda=0,68 \mu \mathrm{m}$ est respectivement de 0,68 et 0,85 .

6. Le rendement énergétique sous un éclairement de $50 \mathrm{~mW} / \mathrm{cm}^{2}$ est de l'ordre de $3 \%$. On voit sur la figure 10 que le point de charge optimum donne $V_{0}=315 \mathrm{mV}, I_{0}=5 \mathrm{~mA}$, soit une résistance optimum de $63 \Omega$ pour une surface de $1 \mathrm{~cm}^{2}$.

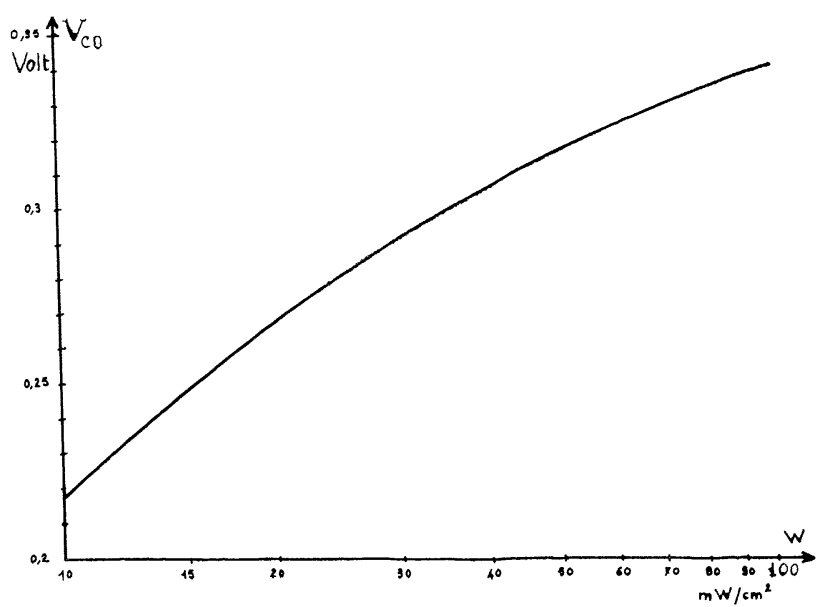

FIG. 8. - Caractéristique $V_{\text {co }}=f(\log W)$.

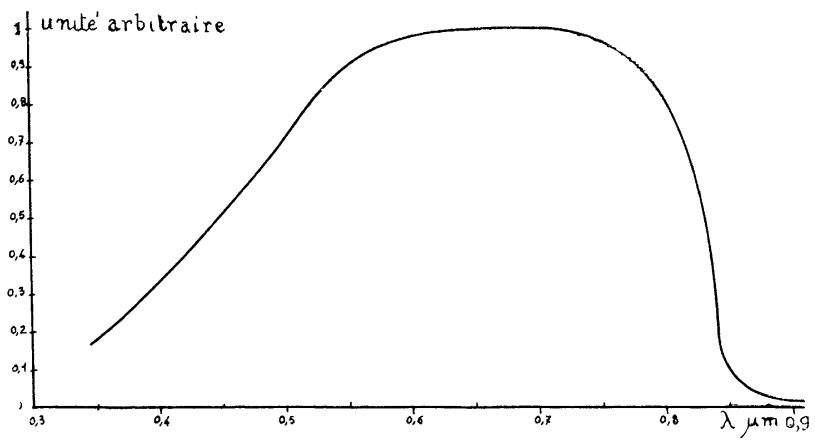

Fig. 9. - Réponse spectrale.

7. Le coefficient de forme $V_{0} I_{0} / V_{\mathrm{oc}} I_{\mathrm{cc}}$ atteint 0,45 .

8. Mesure de l'épaisseur de la zone désertée. Par mesure de capacité de la jonction en polarisation inverse, on détermine des épaisseurs allant de 0,5 à $2 \mu \mathrm{m}$.

d) Traitement thermique. - Nous avons étudié, en fonction de la température, l'évolution de certains paramètres.

Lorsque les photopiles ont un très mauvais rendement, l'évolution de $I_{\mathrm{cc}}, V_{\mathrm{co}}$, et puissance fournie sous un éclairement donné est conforme à la figure 11. Ceci traduit une véritable transformation des cellules.

Malgré de si faibles températures on est tenté de penser à une diffusion du cuivre par exemple.

Lorsque les cellules ont, dès le départ, de honnes caractéristiques, les phénomènes sont d'une autre nature et se prêtent mieux à une interprétation. En général avec de bonnes cellules, avec ou sans CdTe $(i)$, les variations sont beaucoup plus faibles.

Le tableau résume les résultats pour des photopiles avec $\mathrm{Cu}_{2-x} \mathrm{Te}$ évaporé, avec et sans $\mathrm{CdTe}(i)$ et pour un traitement à $130^{\circ} \mathrm{C}$ pendant $1 / 2$ heure.

On est tenté d'expliquer ceci par une diffusion du cuivre de $\mathrm{Cu}_{2-x} \mathrm{Te}$ dans CdTe comme l'avait fait 


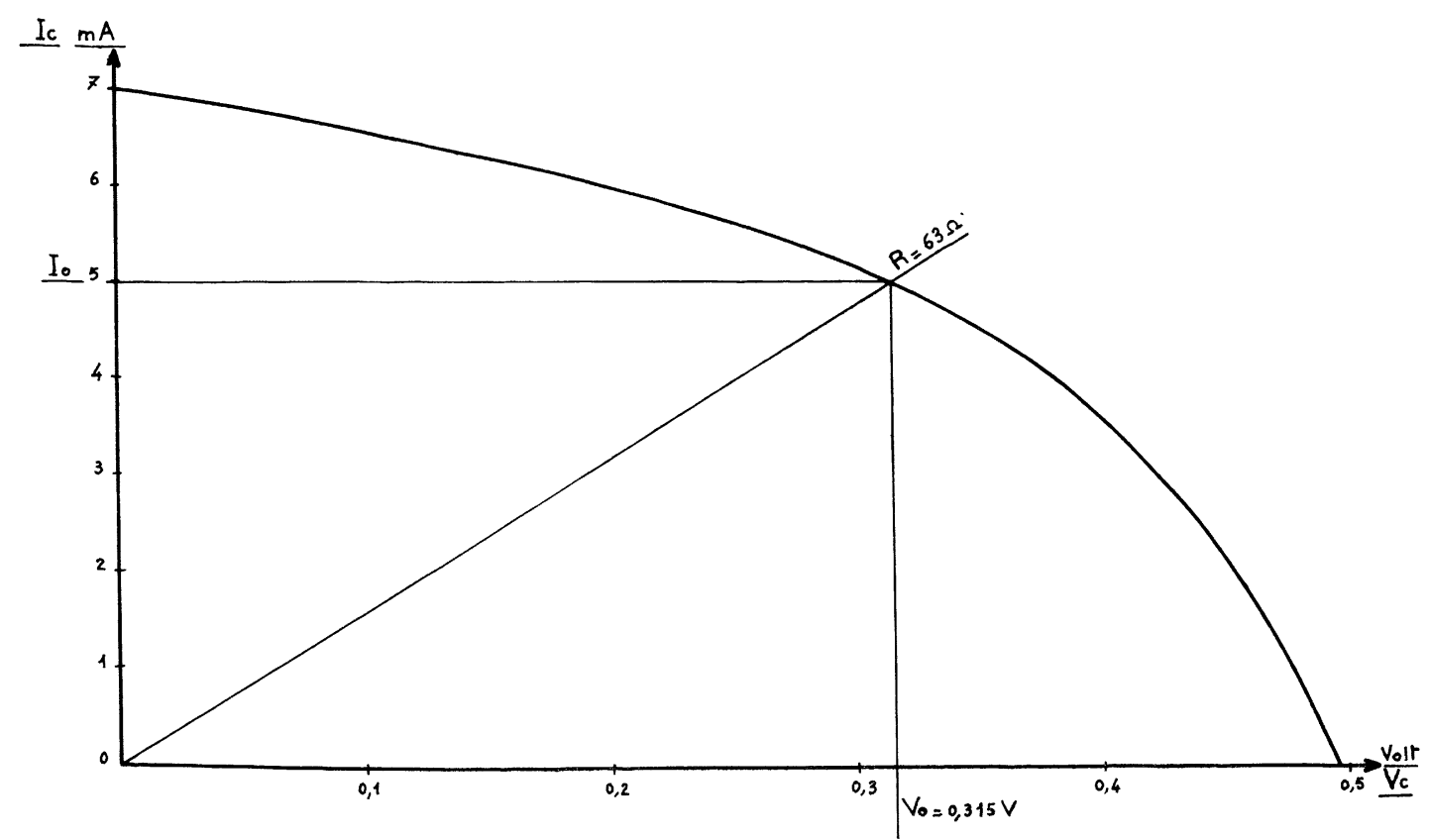

Fig. 10. - Caractéristique $I=f(V) E=50 \mathrm{~mW} / \mathrm{cm}^{2}$.

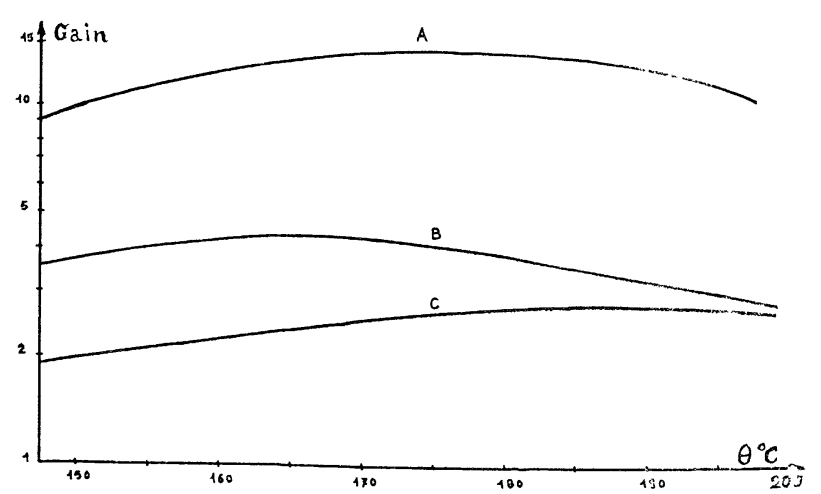

FIG. 11. - Traitement thermique.

$$
\begin{aligned}
\mathrm{A} & =\frac{\text { puissance max. après recuit }}{\text { puissance max. avant recuit }} \\
\mathrm{B} & =\frac{I_{\text {cc }} \text { après recuit }}{I_{\mathrm{c}} \text { avant recuit }} \\
\mathrm{C} & =\frac{V_{\text {co après recuit }}}{V_{\text {co avant recuit }}}
\end{aligned}
$$

\section{Sans CdTe Avec CdTe}

$$
\begin{array}{lll}
\text { Gain } I_{\mathrm{cc}} & -\overline{10} \% & -\overline{5} \% \\
\text { Gain } V_{\mathrm{co}} & +5 \% & +3 \%
\end{array}
$$

J. Bernard [2] travaillant sur CdTe massif. En effet ceci se traduit :
$1^{0}$ par un accroissement de résistance superficielle entraînant une diminution de $I_{\text {cc }}$;

$2^{\circ}$ par un accroissement de $V_{\text {co }}$ par suite d'un dopage $p$ du CdTe $(i)$ ou CdTe $(n)$.

J. Bernard avait d'ailleurs conclu à la formation d'une homojonction en s'appuyant également sur d'autres critères il est vrai ; toutefois si nous opérons une évaporation ultérieure de $\mathrm{Cu}_{2-x} \mathrm{Te}$ destinée à compenser cette perte, $I_{\text {cc }}$ et $V_{\text {co }}$ diminuent et ceci dans un rapport qui est celui correspondant à l'absorption de la couche de $\mathrm{Cu}_{2-x}$ Te ajoutée.

Conclusions. - Par une méthode qui s'éloigne de celle décrite par Cusano en ce qui concerne la réalisation de la couche $p$, nous avons donc pu réaliser des photopiles solaires en couches minces dont les caractéristiques actuelles sont encore modestes; rappelons les :

$V_{\text {co }}: 400$ à $560 \mathrm{mV}$

$J_{\text {cc }}: 7 \mathrm{~mA} / \mathrm{cm}^{2}$ (sous un éclairement de $50 \mathrm{~mW} / \mathrm{cm}^{2}$

Coefficient de forme : 0,45 .

$$
\eta: 3 \% \text {. }
$$

Mais nous savons que nous disposons encore d'un certain nombre de paramètres que nous pourrons faire varier pour accroître ce rendement.

Remerciements. - L'auteur remercie ses collaborateurs ingénieurs et techniciens du groupe photopiles solaires du Laboratoire d'Études et Recherches Générales de R. T. C. (Suresnes) qui ont réalisé et mesuré ces photopiles solaires.

\section{BIBLIOGRAPHIE}

[1] Cusano (D. A.), CdTe Solar Cells and photovoltaic Heterojonctions in II-VI compounds. Solid State Electronics, 1963, 6, 217-232.
[2] Bernard (J.), Thèse 3e cycle, Paris, 14 décembre 1965. 